\title{
Influence of an inert charged Higgs boson on the muon $g-2$ and radiative neutrino masses in a scotogenic model
}

\author{
Chuan-Hung Chen ${ }^{1, *}$ and Takaaki Nomura ${ }^{2, \dagger}$ \\ ${ }^{1}$ Department of Physics, National Cheng-Kung University, Tainan 70101, Taiwan \\ ${ }^{2}$ School of Physics, KIAS, Seoul 02455, Korea
}

(Received 22 March 2019; published 17 July 2019)

\begin{abstract}
A simple extension of Ma's approach in a scotogenic model is studied for the purpose of simultaneously interpreting the neutrino data and the excess of the muon anomalous magnetic moment (muon $g-2$ ). The feasible minimal extension is to add a $Z_{2}$-odd vectorlike lepton doublet to the Ma's model. It is found that in addition to the neutrino data, the strict constraints on the relevant parameters are from the electroweak oblique parameters and the induced lepton-flavor violation processes, such as $\ell_{i} \rightarrow \ell_{j} \gamma$ and $\ell_{i} \rightarrow \ell_{j}^{-} \ell_{j}^{-} \ell_{j}^{+}$. Performing a parameter scan, we numerically demonstrate that when the constraint conditions are satisfied, the muon $g-2$ of $O\left(10^{-9}\right)$ can be achieved, where it can be expected that with a $5 \sigma$ observation, the Muon $g-2$ experiment at Fermilab can observe $a_{\mu} \approx 13.31 \times 10^{-10}$ when the current experiment and the SM errors are reduced by a factor of 4 and 2, respectively. Moreover, the branching ratio of the $\tau \rightarrow \mu \gamma$ decay can match the Belle II sensitivity of $O\left(10^{-9}\right)$ with an integrated luminosity of $50 \mathrm{ab}^{-1}$.
\end{abstract}

DOI: $10.1103 /$ PhysRevD.100.015024

\section{INTRODUCTION}

In addition to the origin of neutrino mass, a clear hint for new physics is the muon anomalous magnetic moment (muon $g-2$ ). The results measured by the E821 experiment at Brookhaven National Lab (BNL) [1] and calculated in the standard model (SM) are, respectively, given as [2]

$$
\begin{aligned}
& a_{\mu}^{\exp }=(11659209.1 \pm 5.4 \pm 3.3) \times 10^{-10}, \\
& a_{\mu}^{\mathrm{SM}}=(11659182.3 \pm 0.1 \pm 3.4 \pm 2.6) \times 10^{-10},
\end{aligned}
$$

where the uncertainties in the SM are from the electroweak, lowest-order hadronic, and higher-order hadronic effects. The difference between the SM and experiment is [2]

$$
\Delta a_{\mu}=a_{\mu}^{\exp }-a_{\mu}^{\mathrm{SM}}=(26.8 \pm 6.3 \pm 4.3) \times 10^{-10},
$$

which indicates a $3.5 \sigma$ deviation. Moreover, the recent theoretical analysis shows a $3.7 \sigma$ deviation [3]. Accordingly, resolutions to the muon $g-2$ excess have been broadly studied in the literature [4-19]. A detailed review of the muon $g-2$ can be found in [20-23].

\footnotetext{
physchen@mail.ncku.edu.tw nomura@kias.re.kr
}

Published by the American Physical Society under the terms of the Creative Commons Attribution 4.0 International license. Further distribution of this work must maintain attribution to the author(s) and the published article's title, journal citation, and DOI. Funded by SCOAP ${ }^{3}$.
The new muon $g-2$ measurements performed in the E989 experiment at Fermilab and the E34 experiment at J-PARC will aim for a precision of 0.14 [24] and $0.10 \mathrm{ppm}$ [25], in which the experimental accuracy can be improved by a factor of 4 and 5 , respectively. If we assume the future experimental and theoretical uncertainties can be, respectively, reduced by a factor of 4 and 2 , it is expected that with a $5 \sigma$ measurement, $\Delta a_{\mu} \approx 13.31 \times 10^{-10}$ can be observed by the Fermilab muon $g-2$ experiment, which has started taking data [26].

It is a highly nontrivial issue to simultaneously generate the neutrino mass at the $10^{-2} \mathrm{eV}$ scale and explain the muon $g-2$ excess in a simple extension of the SM. One of the feasible possibilities to accommodate both phenomena is that both processes can be achieved through the quantum radiative corrections. A known mechanism for a radiative neutrino mass of $O\left(10^{-2}\right) \mathrm{eV}$ is the scotogenic model proposed in [27] (called the "Ma model" in this paper), where the dark matter (DM) candidate can be the lightest inert neutral scalar or the right-handed neutrino $\left(N_{k}\right)$ [27,28].

It is found that the Ma model cannot generate a sufficient $\Delta a_{\mu}$ without an extension. The main reasons are as follows: (i) The lepton anomalous magnetic moment can be generated by the mediation of inert charged Higgs bosons and dark right-handed neutrinos. Since the involved charged leptons are left-handed, to match the chirality of tensor-type dipole operators, the effect is indeed suppressed by $m_{\ell}^{2} / m_{N_{k}}^{2}$. (ii) $a_{\mu}^{\mathrm{NP}}$ induced by a charged Higgs boson at the one-loop level is usually negative [29]. Therefore, in this work, we study whether the neutrino data and $a_{\mu}^{\mathrm{NP}} \sim O\left(10^{-9}\right)$ can be 
accommodated in a scotogenic model when the Ma model is minimally extended.

We find that the feasible minimal extension is to include a $Z_{2}$-odd vectorlike lepton doublet $(X)$. Due to the new dark lepton doublet, the left-handed and right-handed couplings can now appear in the same loop diagram; therefore, the induced $a_{\mu}^{\mathrm{NP}}$ is proportional to $m_{\mu}$, not $m_{\mu}^{2}$. Because more Yukawa couplings are involved, we have the degrees of freedom to make the inert charged-Higgs-boson-induced $a_{\mu}^{\mathrm{NP}}$ positive. Although the inert neutral scalar bosons can also contribute to the muon $g-2$, due to strong cancellation and $m_{\ell}^{2} / m_{N_{k}}^{2}$ suppression, their effects are small and can be neglected. Intriguingly, it will be shown that the proposed model can originate from a larger gauge symmetry, such as $S O(10)$ [30,31].

Since we concentrate the study in the flavor physics, we do not analyze the DM-related physics in this study. The relevant DM analysis can be found in [32-51]. It is worth mentioning that it has been found that in some parameter regions, the imposed $Z_{2}$ symmetry in the original Ma model could be broken when renormalization group equation (RGE) effects are taken into account [40,41,45]. The possible resolutions to the problem can be found in $[39,43,44]$. In addition, we also skip the analysis for the signal search at the LHC, where the related discussions can be found in Refs. [52-60].

In addition to the neutrino physics and muon $g-2$, lepton flavor violation (LFV) processes, such as $\ell_{i} \rightarrow \ell_{j} \gamma$ and $\ell_{i} \rightarrow \ell_{j}^{-} \ell_{j}^{-} \ell_{j}^{+}\left(\ell_{i} \rightarrow 3 \ell_{j}\right)$, can be produced in the extension model $[39,61]$. Additionally, $X$ and $N_{k}$ can together couple through the SM Higgs doublet, so that the electroweak oblique parameters may constrain the related parameters due to the mass splitting within the vectorlike lepton doublet. Hence, it is a challenge to require all related parameters through various combinations to fit the current experimental upper limits. After taking some assumptions based on the $\mu \rightarrow e \gamma$ constraint, 11 new independent parameters are involved. We will show that the 11 free parameters can be accommodated in the model when all constraints from the electroweak oblique parameters, the LFV processes, and the neutrino data are satisfied; and the muon $g-2$ can still reach the level of $10^{-9}$.

When the $\mu \rightarrow e \gamma$ constraint is compromised in the model, indeed, $\tau \rightarrow \mu \gamma$ exerts an important constraint on the parameters, especially those related to the neutrino mass matrix for which we cannot arbitrarily tune the parameters to be small. After scanning the chosen parameter regions, it is found that the branching ratio (BR) for the $\tau \rightarrow \mu \gamma$ decay can be well controlled in the model and that $\operatorname{BR}(\tau \rightarrow \mu \gamma)$ can be as large as the current upper bound of $4.4 \times 10^{-8}$, depending on the values of the involved parameters. With $50 \mathrm{ab}^{-1}$ of data accumulated at the Belle II, the sample of $\tau$ pairs can be increased to approximately $5 \times 10^{10}$, where the sensitivity necessary to observe the LFV $\tau$ decays can reach
$10^{-10}-10^{-9}$ [62]. If Belle II observes $\operatorname{BR}(\tau \rightarrow \mu \gamma)$ at the level of $10^{-9}$, the scotogenic model can provide the interpretation of the observation.

The paper is organized as follows: We briefly introduce the model and the relevant couplings in Sec. II. In Sec. III, we derive the formulas for the neutrino mass matrix, for the $\ell_{i} \rightarrow \ell_{j} \gamma$ decays, for the $\ell_{i} \rightarrow 3 \ell_{j}$ decays, and for the lepton $g-2$. Based on the neutrino oscillation data, we also show the allowed region for each neutrino mass matrix element. The parameter scan and the detailed numerical analysis are shown in Sec. IV. In that section, we also provide a detailed numerical analysis of the relevant phenomena. A summary is given in Sec. V.

\section{MODEL}

In this study, we extend the SM gauge symmetry, including a $Z_{2}$-parity symmetry. In order to generate the neutrino mass through a one-loop radiative mechanism and provide the dark matter candidate, we add three right-handed neutrinos $N_{k}=$ $(1,0)(k=1,2,3)$ and one inert Higgs doublet $H_{I}=(2,1)$ to the SM [27], where both $N_{k}$ and $H_{I}$ are $Z_{2}$-odd states, and numbers in brackets denote the $S U(2)_{L}$ representation and $U(1)_{Y}$ hypercharge, respectively. Using the introduced $N_{k}$ and $H_{I}$, it is found that the muon $g-2$ can be significantly enhanced when a vectorlike lepton doublet $X_{L(R)}=(2,-1)$ is included. Since the heavy lepton doublet has to couple to the $\mathrm{SM}$ leptons and $Z_{2}$-odd particles, i.e., $N_{k}$ and $H_{I}, X_{L(R)}$ must carry the $Z_{2}$ charge. Thus, in addition to $N_{k}$, which is free from the mixing with the SM neutrino [27], in principle, the new neutral lepton $\chi_{L}^{0 C}$ and scalar bosons can be the DM candidate.

\section{A. Yukawa couplings and mass splitting in dark lepton doublet}

The gauge invariant lepton Yukawa couplings under $S U(2)_{L} \times U(1)_{Y} \times Z_{2}$ symmetry can be written as

$$
\begin{aligned}
-\mathcal{L}_{Y}= & y_{i j}^{\ell} \bar{L}_{i} H \ell_{R j}+y_{L i}^{k} \bar{L}_{i} \tilde{H}_{I} N_{k}+y_{R j} \bar{X}_{L} H_{I} \ell_{R j} \\
& +h_{L}^{k} \bar{X}_{L} \tilde{H} N_{k}+\frac{m_{N_{k}}}{2} \overline{N_{k}^{C}} N_{k}+m_{X} \bar{X}_{L} X_{R}+\text { H.c. }
\end{aligned}
$$

where $i, j=1,2,3$ denote the flavor indices; $H^{T}=$ $\left(G^{+},\left(v+h+i G^{0}\right) / \sqrt{2}\right)$ is the SM Higgs doublet and $v$ is the vacuum expectation value (VEV) of $H ; N^{C}=C \gamma^{0} N^{*}$ with $C=i \gamma^{0} \gamma^{2}, \tilde{H}_{(I)}=i \tau_{2} H_{(I)}^{*} ; m_{N}$ and $m_{X}$ are the masses of $N_{R}$ and $X_{L(R)}$, respectively; and the representations of dark $H_{I}$ and $X_{L(R)}$ are given as

$H_{I}=\left(\begin{array}{c}H_{I}^{+} \\ \left(S_{I}+i A_{I}\right) / \sqrt{2}\end{array}\right), \quad X_{L(R)}=\left(\begin{array}{c}\chi^{0} \\ \chi^{-}\end{array}\right)_{L(R)}$.

Since $\chi^{-}$is a $Z_{2}$-odd particle and cannot mix with the SM charged leptons after electroweak symmetry breaking 
(EWSB), the SM charged-lepton masses are still dictated by the first term in Eq. (3). That is, the SM leptons in Eq. (3) can be taken as the physical states after EWSB and their masses can be expressed as $m_{\ell_{i}}=y_{i j}^{\ell} v \delta_{i j} / \sqrt{2}$. In terms of the representation components, the new Yukawa interactions are written as

$$
\begin{aligned}
& -\mathcal{L}_{Y} \supset\left(y_{L i}^{k} \bar{\nu}_{L i} N_{k}+y_{R i} \bar{\ell}_{R i} \chi_{L}^{-}\right) \frac{S_{I}+i A_{I}}{\sqrt{2}}+h_{L}^{k} \overline{\chi_{L}^{0}} N_{k} \frac{v+h}{\sqrt{2}} \\
& +\frac{m_{N_{k}}}{2} \overline{N_{k}^{C}} N_{k}+\left(y_{R i} \overline{\chi_{L}^{0}} \ell_{R i}-y_{L i}^{k} \bar{N}_{k} \ell_{L i}\right) H_{I}^{+} \\
& +m_{X}\left(\overline{\chi_{L}^{0}} \chi_{R}^{0}+\overline{\chi_{L}} \chi_{R}^{-}\right)+\text {H.c. },
\end{aligned}
$$

where $y_{R i}$ and $h_{L}^{k}$ are taken as the real parameters.

It is worth mentioning that the proposed model can arise from a larger gauge group, such as $S O(10)$ grand unified theories (GUTs) [30,31], where the symmetry breaking chain is $S O(10) \rightarrow S U(5) \times U(1)_{\chi} \rightarrow S U(3)_{C} \times S U(2)_{L} \times$ $U(1)_{Y} \times U(1)_{\chi}$. Denoting all fermion representations as the left-handed states, the new lepton doublets $X$ and $X^{c}$ can originate from 10 of $S O(10)$ and can be $(\overline{\mathbf{5}},-2)+$ $(\mathbf{5}, 2)$ in $S U(5) \times U(1)_{\chi}$. If we embed the inert doublet $\tilde{H}_{I}$, the right-handed neutrinos $N_{k}$, and the SM Higgs field in the representations of $\mathbf{1 6} \supset(\overline{\mathbf{5}}, 3), \mathbf{4 5} \supset(\mathbf{1}, 0)$, and 10, the Yukawa interactions $\bar{X}_{L} \tilde{H} N_{k}$ and $\bar{X}_{L} H_{I} \ell_{R}$ can be gauge singlets under the gauge symmetries $10 \times \mathbf{1 0} \times \mathbf{4 5}$ and $10 \times 16 \times 16$, respectively.

Because the SM Higgs doublet $H$ couples to $X_{L, R}$ and $N_{R}$ and $\chi_{L, R}^{0}$ can mix with $N_{R}$ when the electroweak symmetry is broken, the $5 \times 5$ neutral lepton mass matrix in the basis $\left(\chi_{R}^{0}, \chi_{L}^{0 C}, N_{k}\right)$ can be written as

$$
M=\left(\begin{array}{ccc}
0 & m_{X} & \mathbf{0}_{1 \times 3} \\
m_{X} & 0 & v \mathbf{h}_{L} / \sqrt{2} \\
\mathbf{0}_{3 \times 1} & v \mathbf{h}_{L}^{T} / \sqrt{2} & \left(\mathbf{m}_{N}\right)_{3 \times 3}
\end{array}\right),
$$

with $\mathbf{h}_{\mathbf{L}}=\left(h_{L}^{1}, h_{L}^{2}, h_{L}^{3}\right)$ and $\mathbf{m}_{N}=\operatorname{diag}\left(m_{N_{1}}, m_{N_{2}}, m_{N_{3}}\right)$. The symmetric mass matrix can be diagonalized using an orthogonal matrix. With the assumption of $m_{N_{1}}=m_{N_{2}}=$ $m_{N_{3}}=m_{0}$, the eigenvalues of the five Majorana states can be obtained as

$$
\begin{aligned}
m_{1} & \approx-m_{X}-\left(e_{X}-e_{N}\right), \quad m_{2} \approx m_{X}+e_{X}, \\
m_{3(4)} & =m_{0}, \quad m_{5} \approx m_{0}-e_{N}, \\
e_{X} & =\frac{v^{2}}{8\left(m_{X}+m_{0}\right)}\left(\eta_{h} \pm \sqrt{\eta_{h}^{2}+16 \zeta_{h}\left(m_{X}+m_{0}\right)^{2} / v^{2}}\right), \\
\eta_{h} & =\zeta_{h}+4 \frac{m_{N}^{2}-m_{X}^{2}}{v^{2}}, \quad e_{N}=e_{X}-\frac{v^{2} \zeta_{h}}{4\left(m_{X}+m_{0}\right)},
\end{aligned}
$$

where we define $\zeta_{h}=\sum_{k}\left(h_{L}^{k}\right)^{2}$, and $h_{k} \equiv v h_{L}^{k} / \sqrt{2}$ is taken as the perturbative parameters, and the \pm sign in $e_{X}$ can determine what the lightest Majorana particle is, i.e., $\chi_{L}^{0 C}$ or one of $N_{k}$. Note that in order to simplify the analysis for the flavor physics, we set all $m_{N_{k}}$ to be the same although generally this is not necessary. If the DM candidate is the lightest right-handed neutrino $\left(N_{k^{\prime}}\right)$, we can take $m_{N_{k^{\prime}}}$ to be smaller than the others. Since our main target is on the flavor physics, we do not further pursue the DM issue in this work. The relevant discussion can be found in [27,32-48,51]. Using the obtained eigenvalues, the flavor mixing matrix can be approximately formulated as

$$
\mathcal{O}_{X} \approx\left(\begin{array}{ccccc}
\frac{m_{X}}{N_{-X}\left|m_{1}\right|}-\frac{1}{N_{-X}} & \frac{h_{1}}{2 m_{0} N_{-X}} & \frac{h_{2}}{2 m_{0} N_{-X}} & \frac{h_{3}}{2 m_{0} N_{-X}} \\
\frac{m_{X}}{N_{X} m_{2}} & \frac{1}{N_{X}} & -\frac{h_{1}}{N_{X}\left(m_{0}-m_{2}\right)} & -\frac{h_{2}}{N_{X}\left(m_{0}-m_{2}\right)} & -\frac{h_{3}}{N_{X}\left(m_{0}-m_{2}\right)} \\
0 & 0 & \frac{h_{2}}{N_{N_{1}} \sqrt{h_{1}^{2}+h_{2}^{2}}} & -\frac{h_{1}}{N_{N_{1}} \sqrt{h_{1}^{2}+h_{2}^{2}}} & 0 \\
0 & 0 & \frac{h_{1}}{N_{N_{2}} \sqrt{h_{1}^{2}+h_{2}^{2}}} & \frac{h_{2}}{N_{N_{2}} \sqrt{h_{1}^{2}+h_{2}^{2}}} & -\frac{\sqrt{h_{1}^{2}+h_{2}^{2}}}{N_{N_{2}} h_{3}} \\
\frac{m_{X}}{N_{N_{3}} m_{5}} & \frac{1}{N_{N_{3}}} & -\frac{h_{1}}{N_{N_{3}}\left(m_{0}-m_{5}\right)} & -\frac{h_{2}}{N_{N_{3}}\left(m_{0}-m_{5}\right)} & \frac{h_{3}}{N_{N_{3}}\left(m_{0}-m_{5}\right)}
\end{array}\right),
$$

where $N_{a}\left(a=-X, X, N_{k}\right)$ are the normalization factors, which follow $\sum_{i} \mathcal{O}_{X a i}^{2}=1$.

From the results, it can be seen that the mass splitting within the vectorlike lepton doublet can be expressed as $\Delta m_{X}=\left|m_{2}-m_{X}\right| \approx\left|e_{X}\right|$ and that it depends on $v h_{L}^{k} / \sqrt{2}$. This mass splitting contributes to the electroweak oblique parameters, where the current measurements with $U=0$ are given as [2]

$$
S=0.07 \pm 0.08, \quad T=0.10 \pm 0.07
$$

Therefore, the precision measurements of electroweak oblique parameters [63] may constrain $h_{L}^{k}$. In order to consider the constraints, we write the oblique corrections for the vectorlike lepton doublet as $[64,65]$

$$
\begin{aligned}
S= & \frac{1}{\pi}\left[\frac{22 z_{1}+14 z_{2}}{9}-\frac{1}{9} \ln \frac{z_{1}}{z_{2}}+\frac{11 z_{1}+1}{18} f\left(z_{1}\right)\right. \\
& \left.+\frac{7 z_{2}-1}{18} f\left(z_{2}\right)-\sqrt{z_{1} z_{2}}\left(4+\frac{f\left(z_{1}\right)+f\left(z_{2}\right)}{2}\right)\right], \\
T= & \frac{1}{8 \pi s_{W}^{2} c_{W}^{2}}\left[z_{1}+z_{2}-\frac{2 z_{1} z_{2}}{z_{1}-z_{2}} \ln \frac{z_{1}}{z_{2}}\right. \\
& \left.+2 \sqrt{z_{1} z_{2}}\left(\frac{z_{1}+z_{2}}{z_{1}-z_{2}} \ln \frac{z_{1}}{z_{2}}-2\right)\right], \\
f(x)= & -4 \sqrt{4 x-1} \arctan \frac{1}{\sqrt{4 x-1}},
\end{aligned}
$$


with $z_{1}=\left(m_{0}-e_{X}\right)^{2} / m_{Z}^{2} \quad$ and $\quad z_{2}=m_{0}^{2} / m_{Z}^{2}$. Since the $U$ parameter usually is small, we do not explicitly show it.

In the calculations of LFV processes, we need the gauge couplings to the photon and $Z$-gauge boson. The relevant interactions are given as

$$
\begin{aligned}
\mathcal{L}_{V}= & -Q_{\ell} e \bar{\ell} \gamma_{\mu} \ell A^{\mu}-i e\left(H_{I}^{-} \partial_{\mu} H_{I}^{+}-\partial_{\mu}\left(H_{I}^{-}\right) H_{I}^{+}\right) A^{\mu} \\
& -\frac{g}{2 \cos \theta_{W}} \overline{\chi^{0}} \gamma_{\mu} \chi^{0} Z^{\mu}-\bar{\ell} \gamma_{\mu}\left(C_{L}^{\ell} P_{L}+C_{R}^{\ell} P_{R}\right) \ell Z^{\mu} \\
& -i \frac{g \cos 2 \theta_{W}}{2 \cos \theta_{W}}\left(H_{I}^{-} \partial_{\mu} H_{I}^{+}-H_{I}^{+} \partial_{\mu} H_{I}^{-}\right) Z^{\mu},
\end{aligned}
$$

with

$$
C_{L}^{\ell}=\frac{g}{2 \cos \theta_{W}}\left(-1+2 \sin ^{2} \theta_{W}\right), \quad C_{R}^{\ell}=\frac{g \sin ^{2} \theta_{W}}{\cos \theta_{W}} .
$$

\section{B. Scalar potential and gauge couplings to dark sector}

The gauge invariant scalar potential with the $Z_{2}$-parity can be written as $[27,28]$

$$
\begin{aligned}
V\left(H, H_{I}\right)= & \mu^{2} H^{\dagger} H+\lambda_{1}\left(H^{\dagger} H\right)^{2}+m_{I}^{2} H_{I}^{\dagger} H_{I}+\lambda_{2}\left(H_{I}^{\dagger} H_{I}\right)^{2} \\
& +\lambda_{3} H^{\dagger} H H_{I}^{\dagger} H_{I}+\lambda_{4} H^{\dagger} H_{I} H_{I}^{\dagger} H \\
& +\frac{\lambda_{5}}{2}\left[\left(H^{\dagger} H_{I}\right)^{2}+\text { H.c. }\right],
\end{aligned}
$$

where $v=\sqrt{-\mu^{2} / \lambda_{1}}$ with $\mu^{2}<0$ and $m_{h}=\sqrt{v^{2} \lambda_{1} / 2}$ are the same as the SM, and the massive inert Higgs doublet requires $m_{I}^{2}>0$. With $v \approx 246 \mathrm{GeV}$ and $m_{h} \approx 125 \mathrm{GeV}$, we can obtain $\lambda_{1} \approx 0.516$. The masses of $\left(S, A, H^{ \pm}\right)$can be expressed as $[27,28]$

$$
\begin{aligned}
& m_{S_{I}}^{2}=m_{I}^{2}+\lambda_{L} v^{2}, \quad m_{A_{I}}^{2}-m_{S_{I}}^{2}=-\lambda_{5} v^{2}, \\
& m_{H_{I}^{ \pm}}^{2}=m_{I}^{2}+\frac{\lambda_{3}}{2} v^{2}
\end{aligned}
$$

with $\lambda_{L}=\left(\lambda_{3}+\lambda_{4}+\lambda_{5}\right) / 2$. It can be seen that the mass difference between $S_{I}$ and $A_{I}$ is dictated by the $\lambda_{5}$ parameter. We will show that, in addition to the Yukawa couplings, the radiative neutrino mass also depends on the mass difference. If $y_{L i}^{k} \sim O\left(10^{-2}\right)$ are required, $\left|\lambda^{5}\right| \sim 10^{-8}$ is necessary to fit the neutrino mass matrix elements, which are of $O\left(10^{-2}\right) \mathrm{eV}$.

In the model, the DM particle can be the lightest $N_{k}$ or $S_{I}\left(A_{I}\right)$. If we select $S_{I}$ or $A_{I}$ as the DM candidate, in order to escape the constraint from the DM-nucleus scattering, which is generated through the $S_{I} A_{I} Z$ gauge coupling [28], $\left|m_{A_{I}}-m_{S_{I}}\right|$ must have a low limit in order to kinematically suppress the scattering process. Then, $y_{L i}^{k}$ have to be of the order of $10^{-4}-10^{-3}$ to match the neutrino mass matrix elements. As a result, the muon $g-2$ arising from the inert charged Higgs boson is suppressed. Similarly, $\chi^{0}$ cannot be the DM candidate because the gauge coupling $\chi^{0} \chi^{0} Z$ leads a large cross section in the process of DM scattering off the nucleus. Hence, we will concentrate on the case with $m_{S_{I}\left(A_{I}\right), H_{I}^{ \pm}}>m_{0, X} \cdot$

\section{RADIATIVE NEUTRINO MASS, LFV, AND LEPTON $g-2$}

In this section, we derive the formulas for the neutrino mass matrix, the $\ell_{i} \rightarrow \ell_{j} \gamma$ and $\ell_{i} \rightarrow 3 \ell_{j}$ processes, and lepton $g-2$ in the model. Although the original Ma model can provide sizable contributions to the LFV processes, we checked that with $y_{1}^{k *} y_{2}^{k} \sim O\left(10^{-3}\right)$, the BR for $\mu \rightarrow e \gamma$ is of the order of $10^{-15}$, which is 2 orders of magnitude smaller than the current upper limit. Therefore, in the following analysis, we concentrate on the extension effects.

\section{A. Radiative neutrino mass}

The Majorana neutrino mass arisen from a quantum loop in the scotogenic model is sketched in Fig. 1. It can be seen that in addition to the Yukawa couplings, the essential effect is from the $\left(H^{\dagger} H_{I}\right)^{2}$ coupling, which is dictated by the $\lambda_{5}$ parameter. From the couplings in Eqs. (5) and (13), the Majorana neutrino mass matrix elements can be obtained as $[27,66]$

$m_{i j}^{\nu}=\sum_{k} \frac{y_{L i}^{k} y_{L j}^{k}}{2(4 \pi)^{2}} m_{N_{k}}\left[\frac{m_{A_{I}}^{2} \ln \left(m_{A_{I}}^{2} / m_{N_{k}}^{2}\right)}{m_{N_{k}}^{2}-m_{A_{I}}^{2}}-\frac{m_{S_{I}}^{2} \ln \left(m_{S_{I}}^{2} / m_{N_{k}}^{2}\right)}{m_{N_{k}}^{2}-m_{S_{I}}^{2}}\right]$.

It can be found that $m_{i j}^{\nu}$ can be of $O\left(10^{-2}\right) \mathrm{eV}$ when $\sum_{k} y_{L i}^{k} y_{L j}^{k} \sim O\left(10^{-4}-10^{-3}\right)$, and $m_{S_{I}\left(A_{I}\right)} \approx m_{N_{k}} \approx 1 \mathrm{TeV}$ are used.

The mass matrix can be diagonalized by the PontecorvoMaki-Nakagawa-Sakata (PMNS) matrix as

$$
m_{i j}^{\nu}=U_{\mathrm{MNS}}^{*} m_{\nu}^{\mathrm{diag}} U_{\mathrm{MNS}}^{\dagger},
$$

where $m_{\nu}^{\text {diag }}=\operatorname{diag}\left(m_{1}, m_{2}, m_{3}\right)$, and the PMNS matrix can be parametrized as [2]

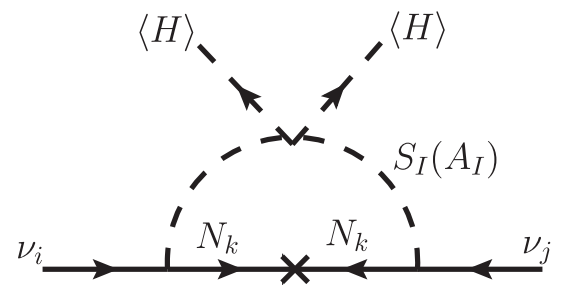

FIG. 1. Feynman diagram for radiative neutrino mass. 


$$
U_{\mathrm{MNS}}=\left(\begin{array}{ccc}
c_{12} c_{13} & s_{12} c_{13} & s_{13} e^{-i \delta} \\
-s_{12} c_{23}-c_{12} s_{23} s_{13} e^{i \delta} & c_{12} c_{23}-s_{12} s_{23} s_{13} e^{i \delta} & s_{23} c_{13} \\
s_{12} s_{23}-c_{12} c_{23} s_{13} e^{i \delta} & -c_{12} s_{23}-s_{12} c_{23} s_{13} e^{i \delta} & c_{23} c_{13}
\end{array}\right) \operatorname{diag}\left(1, e^{i \alpha_{21} / 2}, e^{i \alpha_{31} / 2}\right)
$$

in which $s_{i j} \equiv \sin \theta_{i j}, c_{i j} \equiv \cos \theta_{i j} ; \delta$ is the Dirac $C P$ violating phase; and $\alpha_{21,31}$ are Majorana $C P$ violating phases. Since the mass ordering is still uncertain, the current neutrino data can be shown in terms of the different mass ordering as [2]

$$
\begin{aligned}
\Delta m_{21}^{2} & =(7.53 \pm 0.18) \times 10^{-5} \mathrm{eV}^{2}, \quad \sin ^{2} \theta_{12}=0.307 \pm 0.013 \\
\Delta m_{32}^{2} & =(2.51 \pm 0.05,-2.56 \pm 0.04) \times 10^{-3} \mathrm{eV}^{2} \quad(\mathrm{NO}, \mathrm{IO}), \\
\sin ^{2} \theta_{23} & =\left(0.597_{-0.030}^{+0.024}, 0.592_{-0.030}^{+0.023}\right) \quad(\mathrm{NO}, \mathrm{IO}), \quad \sin ^{2} \theta_{13}=(2.12 \pm 0.08) \times 10^{-2},
\end{aligned}
$$

where $\Delta m_{i j}^{2} \equiv m_{i}^{2}-m_{j}^{2}$, and $\Delta m_{32}^{2}>0$ and $\Delta m_{32}^{2}<0$ denote the normal ordering (NO) and inverted ordering (IO), respectively.

Based on the neutrino oscillation data, the central values of $\theta_{i j}, \delta$, and $\Delta m_{i j}^{2}$ using the global fit can then be obtained as [67]

$$
\begin{aligned}
& \mathrm{NO}: \theta_{12}=34.5^{\circ}, \quad \theta_{23}=47.7^{\circ}, \quad \theta_{13}=8.45^{\circ}, \quad \delta=218^{\circ}, \quad \Delta m_{21}^{2}=7.55 \times 10^{-5} \mathrm{eV}^{2}, \quad \Delta m_{31}^{2}=2.50 \times 10^{-3} \mathrm{eV}^{2} \text {, } \\
& \text { IO: } \theta_{12}=34.5^{\circ}, \quad \theta_{23}=47.9^{\circ}, \quad \theta_{13}=8.53^{\circ}, \quad \delta=281^{\circ}, \quad \Delta m_{21}^{2}=7.55 \times 10^{-5} \mathrm{eV}^{2}, \quad \Delta m_{31}^{2}=-2.42 \times 10^{-3} \mathrm{eV}^{2} \text {, }
\end{aligned}
$$

where $m_{1(3)}=0$ for $\mathrm{NO}(\mathrm{IO})$ are applied, and the Majorana phases are taken to be $\alpha_{21(31)}=0$. Taking the $3 \sigma$ uncertainties, the magnitudes of the Majorana matrix elements in units of $\mathrm{eV}$ for NO and $\mathrm{IO}$ can be, respectively, estimated as

$$
\begin{aligned}
& \left(\begin{array}{lll}
\left|m_{11}^{\nu}\right| & \left|m_{12}^{\nu}\right| & \left|m_{13}^{\nu}\right| \\
\left|m_{21}^{\nu}\right| & \left|m_{22}^{\nu}\right| & \left|m_{23}^{\nu}\right| \\
\left|m_{31}^{\nu}\right| & \left|m_{32}^{\nu}\right| & \left|m_{33}^{\nu}\right|
\end{array}\right)_{\text {NO }} \simeq\left(\begin{array}{ccc}
0.11-0.45 & 0.12-0.82 & 0.12-0.82 \\
0.12-0.82 & 2.4-3.3 & 2.0-2.2 \\
0.12-0.82 & 2.0-2.2 & 2.2-3.1
\end{array}\right) \times 10^{-2} \\
& \left(\begin{array}{lll}
\left|m_{11}^{\nu}\right| & \left|m_{12}^{\nu}\right| & \left|m_{13}^{\nu}\right| \\
\left|m_{21}^{\nu}\right| & \left|m_{22}^{\nu}\right| & \left|m_{23}^{\nu}\right| \\
\left|m_{31}^{\nu}\right| & \left|m_{32}^{\nu}\right| & \left|m_{33}^{\nu}\right|
\end{array}\right)_{\text {IO }} \simeq\left(\begin{array}{ccc}
4.8-5.0 & 0.41-0.65 & 0.39-0.62 \\
0.41-0.65 & 1.9-2.8 & 2.4-2.6 \\
0.39-0.62 & 2.4-2.6 & 2.2-3.1
\end{array}\right) \times 10^{-2} .
\end{aligned}
$$

It can be found that when $\sum_{k} y_{L i^{\prime}}^{k} y_{L j^{\prime}}^{k} \sim 10^{-3}\left(i^{\prime}, j^{\prime}=2,3\right)$ and

$\mathcal{M}_{0}=\frac{m_{0}}{16 \pi^{2}}\left[\frac{m_{A_{I}}^{2} \ln \left(m_{A_{I}}^{2} / m_{0}^{2}\right)}{m_{0}^{2}-m_{A_{I}}^{2}}-\frac{m_{S_{I}}^{2} \ln \left(m_{S_{I}}^{2} / m_{0}^{2}\right)}{m_{0}^{2}-m_{S_{I}}^{2}}\right] \sim \mathrm{eV}$,

$m_{i^{\prime}, j^{\prime}}^{\nu} \sim O\left(10^{-2}\right) \mathrm{eV}$ can then be obtained. We will show that due to the $\mu \rightarrow e \gamma$ constraint, the $y_{L 1}^{k}$-related parameters have to be smaller than $y_{L 2, L 3}^{k}$. Therefore, $m_{1 i}^{\nu}$ are preferred to be smaller than $m_{i^{\prime} j^{\prime}}^{\nu}$; i.e., the model is suitable for the NO case.

\section{B. Radiative $\ell \rightarrow \ell^{\prime} \gamma$ decays}

In the model, the LFV processes can arise from the $S_{I}, A_{I}$, and $H_{I}^{ \pm}$boson exchanges. Since $m_{S_{I}} \approx m_{A_{I}}$ is taken in this work, the $S_{I^{-}}$and $A_{I^{-}}$-induced LFV effects have strong cancellations. Thus, in this study, we concentrate on the inert charged-Higgs-boson effects. The current experimental upper limits on the BR for the relevant LFV processes are shown in Table I.

The Feynman diagrams for the $H_{I}^{ \pm}$-mediated radiative $\ell_{i} \rightarrow \ell_{j} \gamma$ decays are sketched in Fig. 2 , where plot (a) arises from the $H_{I}^{ \pm}$and $\chi^{0}$-fermion loop and plots (b) and (c) are the associated self-energy diagrams, which can be used to remove the ultraviolet divergence. According to the Yukawa couplings in Eq. (5), the effective interactions for $\ell_{i} \rightarrow \ell_{j} \gamma$ can then be obtained as

$$
-\mathcal{L}_{\ell_{i} \rightarrow \ell_{j} \gamma}^{\chi^{0}}=-\frac{e}{2} m_{\ell_{i}} a_{L}^{j i} \bar{\ell}_{j} \sigma_{\mu \nu} P_{L} \ell_{i} F^{\mu \nu}
$$

TABLE I. Current experimental upper limits on the LFV processes.

\begin{tabular}{lcccc}
\hline \hline $\mathrm{LFV}$ & $\mu \rightarrow e \gamma$ & $\mu \rightarrow 3 e$ & $\tau \rightarrow \mu(e) \gamma$ & $\tau \rightarrow 3 \mu(3 e)$ \\
\hline $\mathrm{BR}$ & $4.2 \times 10^{-13}$ & $1.0 \times 10^{-12}$ & $4.4(3.3) \times 10^{-8}$ & $2.1(2.7) \times 10^{-8}$ \\
\hline \hline
\end{tabular}


where the Wilson coefficient and loop integral are given as

$$
\begin{aligned}
a_{L}^{j i} & =\frac{y_{R j}^{*} y_{R i}}{16 \pi^{2} m_{X}^{2}} I_{L}^{\gamma}\left(\frac{m_{H_{I}^{ \pm}}^{2}}{m_{X}^{2}}\right), \\
I_{L}^{\gamma}(a) & =\int_{0}^{1} d x \int_{0}^{x} d y \frac{(x-1)(x-y)}{1-x+a x} .
\end{aligned}
$$

Because $m_{\ell_{j}} \ll m_{\ell_{i}}$, we have neglected the $m_{\ell_{j}}$ effects. Since only right-handed leptons couple to $\chi^{0}$, in order to match the chirality of the dipole operator, a mass insertion in the $\ell_{i}$ leg to flip the $\ell_{i}$ chirality from the right-handed state to the left-handed state becomes necessary. As a result, Eq. (22) is proportional to $m_{\ell_{i}}$, and the left-handed $\ell_{i}$ is involved in the radiative decay. We note that although $\ell_{i} \rightarrow \ell_{j} \gamma$ processes can be induced through the $N_{k}$ mediators, because the associated Yukawa couplings $y_{L j}^{k *} y_{L i}^{k}$ are constrained by the neutrino masses to be of $O\left(10^{-4}-10^{-3}\right)$ [27], we thus neglect their contributions.

In addition to the $H_{I}^{ \pm}-\chi^{0}$ loops shown in Figs. 2(a)-2(c), the $\ell_{i} \rightarrow \ell_{j} \gamma$ can be generated by Fig. 2(d), where the diagram involves the mixing of $\chi^{0}$ and $N_{k}$, where the mixing occurs through the VEV of the SM Higgs field, i.e., $v h_{L}^{k} / \sqrt{2}$. Because $N_{k}$ and $\chi^{0}$ have been massive particles before EWSB, it is more convenient to use the weak eigenstates of $N_{k}$ and $\chi^{0}$ to estimate Fig. 2(d). Accordingly, the effective interactions for $\ell_{i} \rightarrow \ell_{j} \gamma$ can be written as

$$
-\mathcal{L}_{\ell_{i} \rightarrow \ell_{j} \gamma}^{N \chi^{0}}=-\frac{e}{2} m_{\ell_{i}} \bar{\ell}_{j} \sigma_{\mu \nu}\left(b_{L}^{j i} P_{L}+b_{R}^{j i} P_{R}\right) \ell_{i} F^{\mu \nu}
$$

where the Wilson coefficients are obtained as

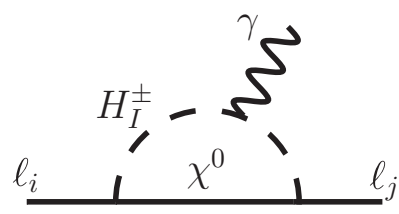

(a)

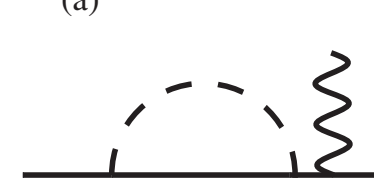

(c)

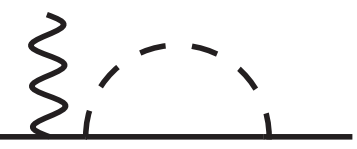

(b)

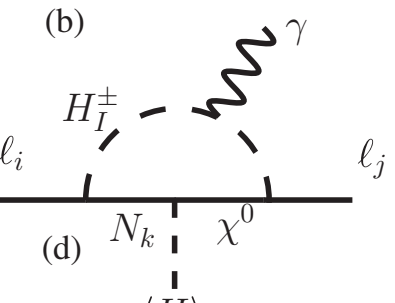

FIG. 2. $\quad \ell_{i} \rightarrow \ell_{j} \gamma$ mediated by the inert charged Higgs boson and the $\chi^{0}$ fermion, where plot (d) includes the mixing effect between $N_{k}$ and $\chi^{0}$.

$$
\begin{aligned}
b_{L}^{j i} & =\frac{1}{16 \pi^{2}} \frac{v y_{R j}^{*} \xi_{L i}}{\sqrt{2} m_{\ell_{i}} m_{X}^{2}} I_{N_{k} \chi^{0}}^{\gamma}\left(\frac{m_{N_{k}}^{2}}{m_{X}^{2}}, \frac{m_{H_{I}^{ \pm}}^{2}}{m_{X}^{2}}\right), \\
b_{R}^{j i} & =\frac{1}{16 \pi^{2}} \frac{v \xi_{L j}^{*} y_{R i}}{\sqrt{2} m_{\ell_{i}} m_{X}^{2}} I_{N_{k} \chi^{0}}^{\gamma}\left(\frac{m_{N_{k}}^{2}}{m_{X}^{2}}, \frac{m_{H_{I}^{ \pm}}^{2}}{m_{X}^{2}}\right), \\
I_{N \chi^{0}}^{\gamma}(a, b) & =\int_{0}^{1} d x \int_{0}^{x} d y \frac{2(1-y) y-y^{2}}{1-(1-a) x-(a-b) y} .
\end{aligned}
$$

Since the $h_{L}^{k}$ parameters always appear to be associated with $y_{L i}^{k}$, we define the independent $\xi_{L i}=y_{L i}^{k} h_{L}^{k}$ parameters to combine the $h_{L}^{k}$ and $y_{L i}^{k}$ effects. In the numerical analysis, we take all $m_{N_{k}}$ to be the same; therefore, $y_{L i}^{k} h_{L}^{k}$ can be read as the sum of all $k$. Because the left- and righthanded lepton couplings appear in Fig. 2(d) at the same time, it can be seen that the mass insertion in the $\ell_{i}$ leg is not necessary. In order to combine this effect with that arisen from the $\chi^{0}$ loop, the $m_{\ell_{i}}$ factor is shown in Eq. (24); as a result, $b_{L, R}^{j i}$ are $1 / m_{\ell_{i}}$ dependent. Combining Eqs. (22) and (24), the BR for $\ell_{i} \rightarrow \ell_{j} \gamma$ can be expressed as

$$
\operatorname{BR}\left(\ell_{i} \rightarrow \ell_{j} \gamma\right)=\tau_{\ell_{i}} \frac{\alpha m_{\ell_{i}}^{5}}{4}\left(\left|T_{L}^{j i}\right|^{2}+\left|T_{R}^{j i}\right|^{2}\right),
$$

with $\alpha=e^{2} / 4 \pi$, and

$$
T_{L}^{j i}=a_{L}^{j i}+b_{L}^{j i}, \quad T_{R}^{j i}=b_{R}^{j i}
$$

$$
\text { C. } \ell_{i} \rightarrow 3 \ell_{j} \text { decays }
$$

The $\ell_{i} \rightarrow 3 \ell_{j}$ decays in the model can arise from the photon-penguin diagrams, e.g., Fig. 2 with the off-shell photon, the $Z$-penguin diagrams, and the box diagrams. We show each decay amplitude as follows: For the photonpenguin diagrams, we write the decay amplitude as

$$
\begin{aligned}
& M\left(\ell_{i} \rightarrow 3 \ell_{j}\right)^{\gamma} \\
& =e^{2} \bar{u}_{j}\left(k_{1}\right)\left[C_{R}^{\gamma i} \gamma_{\mu} P_{R}+\frac{m_{\ell_{i}}}{k^{2}} i \sigma_{\mu \nu} k^{\nu}\left(T_{L}^{j i} P_{L}+T_{R}^{j i} P_{R}\right)\right] u_{i}(p) \\
& \quad \times \bar{u}_{j}\left(k_{2}\right) \gamma^{\mu} v_{j}\left(k_{3}\right)-\left(k_{1} \leftrightarrow k_{2}\right)
\end{aligned}
$$

where $C_{R}^{\gamma j i}$ from Fig. 2(a) is given as

$$
C_{R}^{\gamma i j}=\frac{y_{R i} y_{R j}^{*}}{16 \pi^{2} m_{X}^{2}} I_{1}\left(\frac{m_{H_{I}^{ \pm}}^{2}}{m_{X}^{2}}\right), \quad I_{1}(a)=\int_{0}^{1} d x \frac{x}{1-x+a x} .
$$

Although Fig. 2(d) can also generate vectorial currentcurrent interaction, since its numerical contribution is at least 1 order of magnitude smaller than $C_{R}^{\gamma j i}$, we have ignored its contribution. Using the results in $[68,69]$, the $\mathrm{BR}$ for $\ell_{i} \rightarrow 3 \ell_{j}$ induced by the photon-penguin can be expressed as 


$$
\begin{aligned}
\operatorname{BR}\left(\ell_{i} \rightarrow 3 \ell_{j}\right)^{\gamma}= & \tau_{\ell_{i}} \frac{\alpha^{2} m_{\ell_{i}}^{5}}{32 \pi}\left[\left|C_{R}^{\gamma j i}\right|^{2}-4 \operatorname{Re}\left(C_{R}^{\gamma j i} T_{L}^{j i *}\right)\right. \\
& \left.+\left(\left|T_{L}^{j i}\right|^{2}+\left|T_{R}^{j i}\right|^{2}\right)\left(\frac{16}{3} \ln \frac{m_{\ell_{i}}}{m_{\ell_{j}}}-\frac{22}{3}\right)\right],
\end{aligned}
$$

where $\tau_{\ell_{i}}$ denotes the $\ell_{i}$ lifetime.

The lepton-flavor changing $\ell_{i} \rightarrow \ell_{j} Z$ can be induced by the $Z$-penguin diagrams. In addition to being the same diagrams shown in Fig. 2 but using the $Z$-boson instead of the photon, the $Z$-boson can also be emitted from $\chi^{0}$, as shown in Figs. 2(a) and 2(d). It is found that the $\ell_{i} \rightarrow \ell_{j} Z$ decays arisen from Figs. 2(a)-2(c) are suppressed by $m_{\ell_{i}} m_{\ell_{j}} / m_{X}^{2}$, where the same results are also shown in the $N_{k}$ fermion loop obtained in [61]. If we apply the approximation with $m_{\ell_{j}} \approx 0$, their contributions can be neglected. Thus, the dominant effects indeed are from diagrams related to Fig. 2(d), and the induced effective interactions can be written as

$$
-\mathcal{L}_{\ell_{i} \rightarrow \ell_{j} Z}^{N \chi^{0}}=\bar{\ell}_{j} \gamma_{\mu}\left(C_{L}^{Z j i} P_{L}+C_{R}^{Z j i} P_{R}\right) \ell_{i} Z^{\mu},
$$

where the $C_{L, R}^{Z j i}$ coefficients are given as

$$
\begin{aligned}
C_{L}^{Z j i}= & \frac{\xi_{L j}^{*} y_{R i}}{16 \pi^{2}} \frac{g}{2 \cos \theta_{W}} \frac{v m_{\ell_{i}}}{\sqrt{2} m_{X}^{2}} I_{N \chi^{0}}^{Z}\left(\frac{m_{N_{k}}^{2}}{m_{X}^{2}}, \frac{m_{H_{I}^{ \pm}}^{2}}{m_{X}^{2}}\right), \\
C_{R}^{Z j i}= & \frac{y_{R j}^{*} \xi_{L i}}{16 \pi^{2}} \frac{g}{2 \cos \theta_{W}} \frac{v m_{\ell_{i}}}{\sqrt{2} m_{X}^{2}} I_{N \chi^{0}}^{Z}\left(\frac{m_{N_{k}}^{2}}{m_{X}^{2}}, \frac{m_{H_{I}^{ \pm}}^{2}}{m_{X}^{2}}\right), \\
I_{N \chi^{0}}^{Z}(a, b)= & \int_{0}^{1} d x \int_{0}^{x} d y \int_{0}^{y} d z \\
& \times \frac{z}{[1-(1-a) x-(a-b) z]^{2}} .
\end{aligned}
$$

From the result, the decay amplitude for $\ell_{i} \rightarrow 3 \ell_{j}$ through the $Z$-penguin can be expressed as

$$
\begin{aligned}
M\left(\ell_{i} \rightarrow 3 \ell_{j}\right)^{Z}= & \frac{1}{m_{Z}^{2}} \bar{u}_{j}\left(k_{1}\right) \gamma_{\mu}\left(C_{L}^{Z j i} P_{L}+C_{R}^{Z j i} P_{R}\right) u_{i}(p) \\
& \times \bar{u}_{j}\left(k_{2}\right) \gamma^{\mu}\left(C_{L}^{\ell} P_{L}+C_{R}^{\ell} P_{R}\right) v_{j}\left(k_{3}\right) \\
& -\left(k_{1} \leftrightarrow k_{2}\right) .
\end{aligned}
$$

Accordingly, the $\mathrm{BR}$ for $\ell_{i} \rightarrow 3 \ell_{j}$ can be obtained as $[68,69]$

$$
\begin{aligned}
\operatorname{BR}\left(\ell_{i} \rightarrow 3 \ell_{j}\right)^{Z}= & \tau_{\ell_{i}} \frac{\alpha^{2} m_{\ell_{i}}^{5}}{32 \pi}\left[\frac{2}{3}\left(\left|F_{L L}\right|^{2}+\left|F_{R R}\right|^{2}\right)\right. \\
& \left.+\frac{1}{3}\left(\left|F_{L R}\right|^{2}+\left|F_{R L}\right|^{2}\right)\right]
\end{aligned}
$$

where $F_{L L, R R}$ and $F_{L R, R L}$ are defined as

$$
\begin{array}{ll}
F_{L L}=\frac{C_{L}^{Z j i} C_{L}^{\ell}}{g^{2} \sin ^{2} \theta_{W} m_{Z}^{2}}, & F_{R R}=\frac{C_{R}^{Z j i} C_{R}^{\ell}}{g^{2} \sin ^{2} \theta_{W} m_{Z}^{2}}, \\
F_{L R}=\frac{C_{L}^{Z j i} C_{R}^{\ell}}{g^{2} \sin ^{2} \theta_{W} m_{Z}^{2}}, & F_{R L}=\frac{C_{R}^{Z j i} C_{L}^{\ell}}{g^{2} \sin ^{2} \theta_{W} m_{Z}^{2}} .
\end{array}
$$

The box diagrams mediated by $H_{I}^{ \pm}$and $\chi^{0}$ for $\ell_{i} \rightarrow 3 \ell_{j}$ are shown in Fig. 3. Although the box diagrams mediated by $H_{I}^{ \pm}$and $N_{k}$ can also contribute to the $\ell_{i} \rightarrow 3 \ell_{j}$ decays, because the involved couplings are constrained by the neutrino masses, their effects can be neglected. In addition, there are strong cancellations between the $S_{I}-S_{I}\left(A_{I}-A_{I}\right)$ and $S_{I}\left(A_{I}\right)-A_{I}\left(S_{I}\right)$ box diagrams, so we also ignore the inert scalar and pseudoscalar contributions. Hence, the decay amplitude for $\ell_{i} \rightarrow 3 \ell_{j}$ from Fig. 3 can be obtained as

$$
\begin{aligned}
M\left(\ell_{i} \rightarrow 3 \ell_{j}\right)^{\mathrm{Box}} & =C_{R}^{B j i} \bar{u}_{j}\left(k_{1}\right) \gamma_{\mu} P_{R} u_{i}(p) \bar{u}_{j}\left(k_{2}\right) \gamma^{\mu} P_{R} v_{i}\left(k_{3}\right), \\
C_{R}^{B j i} & =\frac{1}{16 \pi^{2} m_{X}^{2}} y_{R j}^{*} y_{R j} y_{R j}^{*} y_{R i} I_{B}\left(\frac{m_{H_{I}^{ \pm}}^{2}}{m_{X}^{2}}\right) \\
I_{B}(a) & =\int_{0}^{1} d x \frac{x(1-x)}{1-x+a x} .
\end{aligned}
$$

The BR can be found as $[68,69]$

$$
\operatorname{BR}\left(\ell_{i} \rightarrow 3 \ell_{j}\right)^{\mathrm{Box}}=\tau_{\ell_{i}} \frac{m_{\ell_{i}}^{5}}{512 \pi^{3}} \frac{\left|C_{R}^{B j i}\right|^{2}}{6} .
$$

\section{Lepton anomalous magnetic dipole moment}

It is known that the lepton $g-2$ originates from the radiative quantum corrections, where the form factors associated with the quantum effects can be written as

$$
\Gamma^{\mu}=\bar{\ell}\left(p^{\prime}\right)\left[\gamma^{\mu} F_{1}\left(k^{2}\right)+\frac{i \sigma^{\mu \nu} k_{\nu}}{2 m_{\ell}} F_{2}\left(k^{2}\right)\right] \ell(p) .
$$

The lepton $g-2$ can then be defined as

$$
a_{\ell}=\frac{g_{\ell}-2}{2}=F_{2}(0) .
$$

Using this definition, it can be seen that the lepton $g-2$ induced by Figs. 2(a)-2(c) are suppressed by $m_{\ell}^{2} / m_{X}^{2}$, whereas $a_{\ell}$ generated by Fig. 2(d) is dictated by

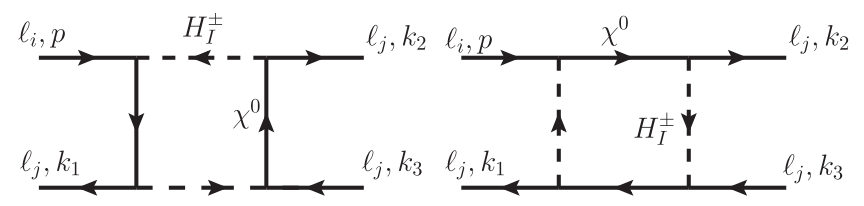

FIG. 3. Box diagrams mediated by $H_{I}^{ \pm}$and $\chi^{0}$ for the $\ell_{i} \rightarrow 3 \ell_{j}$ decays. 
$m_{\ell} / m_{X} \cdot v h_{L}^{k} / m_{X}$. Thus, the dominant lepton $g-2$ in the model can be obtained as

$$
a_{\ell}=-\frac{\operatorname{Re}\left(\xi_{L \ell}^{*} y_{R \ell}\right)}{16 \pi^{2}} \frac{\sqrt{2} m_{\ell} v}{m_{X}^{2}} I_{N_{k} \chi^{0}}^{\gamma}\left(\frac{m_{N_{k}}^{2}}{m_{X}^{2}}, \frac{m_{H_{I}^{ \pm}}^{2}}{m_{X}^{2}}\right) .
$$

Although $a_{\mu}$ is associated with $\sum_{k} y_{L 2}^{k} h_{L}^{k}$, which are related to the neutrino masses, the muon $g-2$ can still be enhanced to $10^{-9}$ if $\xi_{L 2} \sim O(0.01)$ and $y_{R 2} \sim O(1)$ are allowed. In order to satisfy the strict constraints from the $\mu \rightarrow \ell \gamma$ and $\mu \rightarrow 3 e$ decays, we can take the related Yukawa couplings, e.g., $\xi_{L 1}$ and $y_{R 1}$, to be small. Then, the electron $g-2$ is far below the current experimental accuracy in the model.

Before analyzing the relevant phenomena in detail, we roughly estimate the BRs for $\ell_{i} \rightarrow \ell_{j} \gamma$ and the BRs for $\ell_{i} \rightarrow 3 \ell_{j}$, which individually arise from the photonpenguin, $Z$-penguin, and box diagrams. For illustration, a benchmark for the relevant parameters is taken as follows:

$$
\begin{aligned}
& \xi_{L 1}=-10^{-6}, \quad \xi_{L 2}=-0.05, \quad \xi_{L 3}=0.02, y_{R 1}=0.5 \times 10^{-4}, \\
& y_{R 2}=1, \quad y_{R 3}=0.5, \quad m_{X\left(N_{k}\right)}=1 \mathrm{TeV}, \quad m_{H_{I}^{ \pm}}=1.1 \mathrm{TeV},
\end{aligned}
$$

where these parameter values have been taken in such a way that the current upper bounds of the LFV processes shown in Table I are satisfied and $a_{\mu} \sim O\left(10^{-9}\right)$ is achieved. As a result, the corresponding values for $\operatorname{BR}\left(\ell_{i} \rightarrow \ell_{j} \gamma\right), \operatorname{BR}\left(\ell_{i} \rightarrow 3 \ell_{j}\right)$, and $a_{\ell}$ are obtained as

$$
\begin{aligned}
& \quad \operatorname{BR}(\mu \rightarrow e \gamma), \operatorname{BR}(\tau \rightarrow \mu \gamma)) \\
& \quad=\left(4.24 \times 10^{-13}, 3.47 \times 10^{-8}\right), \\
& \quad \operatorname{BR}(\mu \rightarrow 3 e)^{\gamma, Z, \operatorname{Box}} \\
& \quad=\left(2.62 \times 10^{-16}, 9.09 \times 10^{-28}, 6.37 \times 10^{-35}\right), \\
& \operatorname{BR}(\tau \rightarrow 3 \mu)^{\gamma, Z, \operatorname{Box}} \\
& \quad=\left(8.64 \times 10^{-10}, 7.09 \times 10^{-18}, 1.87 \times 10^{-10}\right), \\
& a_{e, \mu, \tau} \\
& \quad=\left(9.15 \times 10^{-21}, 9.24 \times 10^{-10},-3.13 \times 10^{-9}\right) .
\end{aligned}
$$

From the simple analysis, it can be clearly seen that in order to obtain $a_{\mu}$ of $O\left(10^{-9}\right)$, the values of the associated parameters have to be $\left|\xi_{L 2}\right| \sim O(0.01)$ and $y_{R 2} \sim O(1)$. Then, $\mu \rightarrow e \gamma$ inevitably gives a strict constraint on the $\xi_{L 1}$ and $y_{R 1}$ parameters. If $y_{L 1}^{k}$ are of the order of $10^{-3}-10^{-2}$, which are the typical magnitudes for explaining the neutrino data with $\lambda_{5} \ll 1$ [27], the result of $\xi_{L 1} \sim$ $O\left(10^{-6}\right)$ or $\xi_{L 1} \approx 0$ has to rely on the cancellation in $\sum_{k} y_{L 1}^{k} h_{L}^{k}$. Because $\xi_{L 1}, y_{R 1} \ll 1$, the contributions to $\mu \rightarrow$ $3 e$ from the $Z$-penguin and box diagrams are negligible. For $\tau \rightarrow 3 \mu$ decay, the $Z$-penguin contribution is still negligible; however, the box-diagram contribution is somewhat larger and is a factor of 5 smaller than the photonpenguin contribution. Based on these results, it is sufficient to only consider the photon-penguin diagram effects when studying the $\mu \rightarrow 3 e$ and $\tau \rightarrow 3 \mu$ decays. In addition, due to $\xi_{L 1} \ll 1$, it indicates $y_{L 1}^{k}<y_{L 2, L 3}^{k}$. Accordingly, we take the NO case for the neutrino mass matrix in the numerical analysis.

\section{NUMERICAL ANALYSIS}

According to previous analysis, it was found that the neutrino mass, the LFV, and the lepton $g-2$ share some common parameters; however, the correlated parameters appearing in the different phenomena have different forms. From Eq. (20), it is known that we need $\sum_{k} y_{L i^{\prime}}^{k} y_{L j^{\prime}}^{k} \sim 10^{-3}$ and $\sum_{k} y_{L 1}^{k} y_{L i}^{k} \sim 10^{-4}$ to fit the neutrino mass matrix for the NO case. From Eq. (41), it is seen that we need $\xi_{L 1} \approx 0$ and $\xi_{L 2} \sim O(0.01)$ to satisfy the LFV constraints and to explain the muon $g-2$ excess; that is, different lepton flavor Yukawa couplings $y_{L i}^{k}$ should be different in terms of their signs and in sizes. In order to show that the scotogenic model can accommodate the relevant phenomena in the same parameter spaces, in this section, we numerically demonstrate that the accommodation can be achieved in the model.

\section{A. Allowed parameter spaces from the parameter scan}

Since the $\xi_{L i}$ parameters are combined by $h_{L}^{k}$ and $y_{L i}^{k}$, we first study the limit on $h_{L}^{k}$. As discussed earlier, the mass splitting in a vectorlike lepton doublet is $\Delta m_{X}=\left|e_{X}\right|$, and the direct bound is from the electroweak oblique parameters $S$ and $T$. Using the results in Eq. (10), it can be seen that $S$ is far smaller than the current measurement. For instance, with $\zeta_{h}=1$, we obtain $S \approx 8 \times 10^{-3}$; that is, the $S$ parameter cannot constrain the $\zeta_{h}$ parameter. In order to understand the constraint from the $T$ parameter, we show $T$ as a function of $\zeta_{h}$ in Fig. 4(a), where the dashed lines denote the experimental central value with $0,1,2$, and $3 \sigma$ errors, and $m_{X}=m_{N_{k}}=1 \mathrm{TeV}$ and the positive sign in $e_{X}$ are used. From the plot, it can be seen that $T$ linearly depends on $\zeta_{h}$. If we take $3 \sigma$ as the maximum value of $T$, we obtain $\zeta_{h}<0.3$. Moreover, we show $\left|e_{X}\right|$ as a function of $\zeta_{h}$ in Fig. 4(b), where the vertical dashed line corresponds to the $T$ parameter with $3 \sigma$ errors. From the result, it can be found that the maximum value of $\left|e_{X}\right|$ is around $68 \mathrm{GeV}$. Our result is consistent with that obtained in [65]. According to the result, when $h_{L}^{1} \sim h_{L}^{2} \sim h_{L}^{3}$, the upper limit of each parameter then is $h_{L}^{k} \sim 0.3$.

We next numerically show that $\zeta_{h}<0.3, \xi_{L 1}=0$, $\xi_{L 2} \sim O(0.01)$, and that the $m_{i j}^{\nu}$ values shown in Eq. (20) can be accommodated in the model. We note that because $\xi_{L 1} \ll 1$, we use $\xi_{L 1}=0$ in the numerical analysis. From $\xi_{L 1}=0$, we can set 

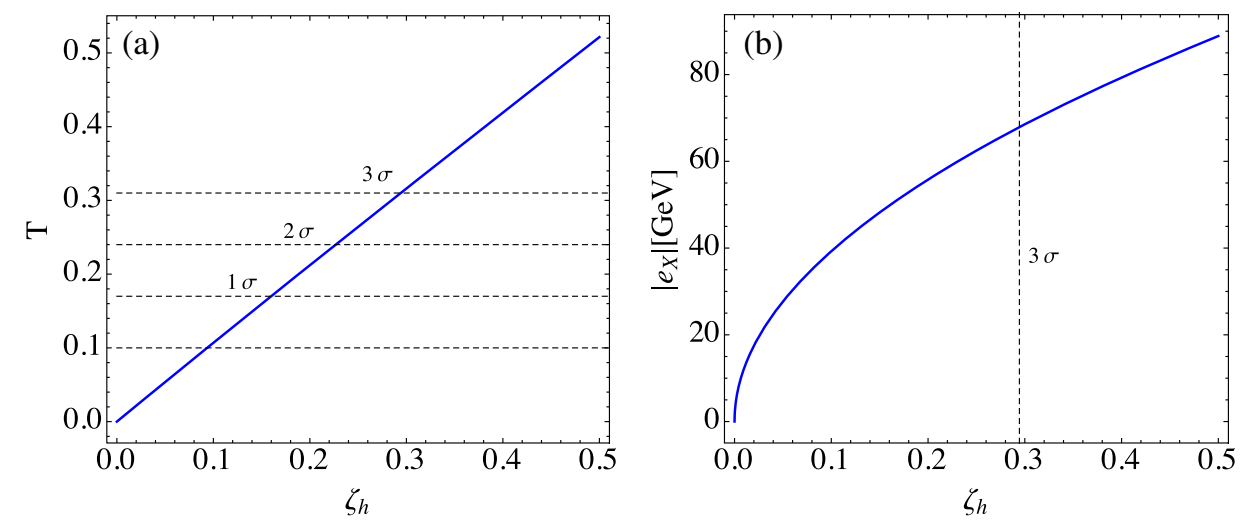

FIG. 4. (a) $T$ parameter as a function of $\zeta_{h}=\sum_{k}\left(h_{L}^{k}\right)^{2}$, where the dashed lines are the experimental central value with 0 , 1, 2, and $3 \sigma$. (b) Mass splitting $\left(e_{X}\right)$ within a vectorlike lepton doublet as a function of $\zeta_{h}$, where the vertical dashed line corresponds to the $T$ parameter with $3 \sigma$ errors.

$$
y_{L 1}^{1}=-\frac{h_{L}^{2}}{h_{L}^{1}} y_{L 1}^{2}-\frac{h_{L}^{3}}{h_{L}^{1}} y_{L 1}^{3} .
$$

To find the allowed parameter spaces, we scan the remaining 11 free parameters in the regions chosen as

$$
h_{L}^{k}=[-0.55,0.55], \quad y_{L i}^{k}=[-10,10] \times 10^{-2} .
$$

In the calculations, we fix $m_{0}=1 \mathrm{TeV}$ and $m_{S_{I}} \approx m_{A_{I}} \approx$ $1.1 \mathrm{TeV}$. In addition, the value of $\left|m_{A_{I}}-m_{S_{I}}\right|$ is taken to fit $\mathcal{M}_{0} \approx 6.12 \times 10^{-9} \mathrm{GeV}$. From the $\left|m_{i j}^{\nu}\right|$ values shown in Eq. (20), the corresponding ranges for $Y_{i j} \equiv\left|\sum_{k} y_{L i}^{k} y_{L j}^{k}\right|$ can then be written as

$$
\begin{aligned}
& Y_{11} \approx(1.80-7.35) \times 10^{-4}, \quad Y_{12} \approx(1.96-13.39) \times 10^{-4}, \\
& Y_{13} \approx Y_{12}, \quad Y_{22} \approx(3.92-5.39) \times 10^{-3}, \\
& Y_{23} \approx(3.27-3.59) \times 10^{-3}, \quad Y_{33} \approx(3.59-5.06) \times 10^{-3} .
\end{aligned}
$$

Using $5 \times 10^{8}$ random sampling points and the chosen scan ranges in Eq. (44), we show the correlation between

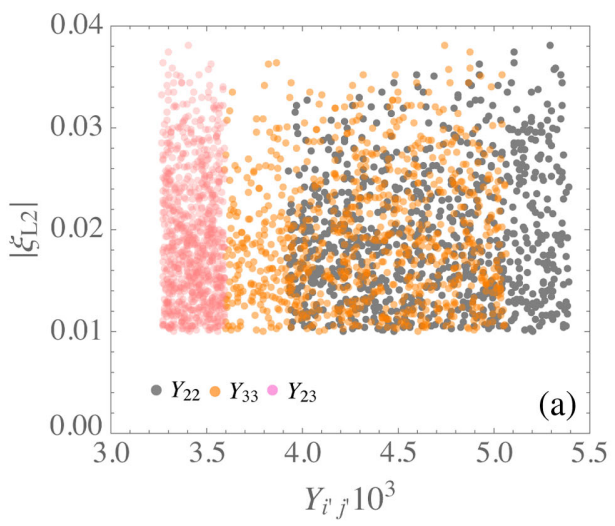

$\left|\xi_{L 2}\right|$ and $Y_{22,33,23}$ in Fig. 5(a), where $\left|\xi_{L 2}\right|>0.01$, $\zeta_{h}<0.3$, and the ranges in Eq. (45) are satisfied. The correlation between $\left|\xi_{L 2}\right|$ and $Y_{1 i}$ is shown in Fig. 5(b). From the analysis, it can be seen that $0.01<\left|\xi_{L 2}\right|<0.04$ can have a good match with $Y_{i j}$, which are determined by the neutrino data.

As mentioned earlier, $\mu \rightarrow e \gamma$ gives a strong constraint on the $\xi_{L 1}$ and $y_{R 1}$ parameters; therefore, a simple way to comply with the requirement is to take $\xi_{L 1}=0$ and $y_{R 1}=0$. However, even so, the $\tau \rightarrow \mu \gamma$ decay may play an important role in constraining the parameters, where from Eqs. (23) and (25), the related parameters are $y_{R 2}^{*} y_{R 3}$, $y_{R 2}^{*} \xi_{L 3}$, and $\xi_{L 2}^{*} y_{R 3}$. If we take the limit with $y_{R 3}=0$, the BR for $\tau \rightarrow \mu \gamma$ does not vanish due to the $y_{R 2}^{*} \xi_{L 3}$ effect. Since $\xi_{L 3}$ is a combination of $h_{L}^{k}$ and $y_{L 3}^{k}$, which are correlated with $\xi_{L 2}$, the $T$ parameter, and the neutrino mass matrix, we cannot arbitrarily tune $\xi_{L 3}$ to be small. In order to see if $\xi_{L 3}$ can be small when the oblique $T$ parameter and neutrino data are satisfied, we show the correlation between $\left|\xi_{L 2}\right|$ and $\left|\xi_{L 3}\right|$ in Fig. 6, where the conditions used to determine the parameter values are the same as those shown in Fig. 5. From the result, it can clearly be seen that when

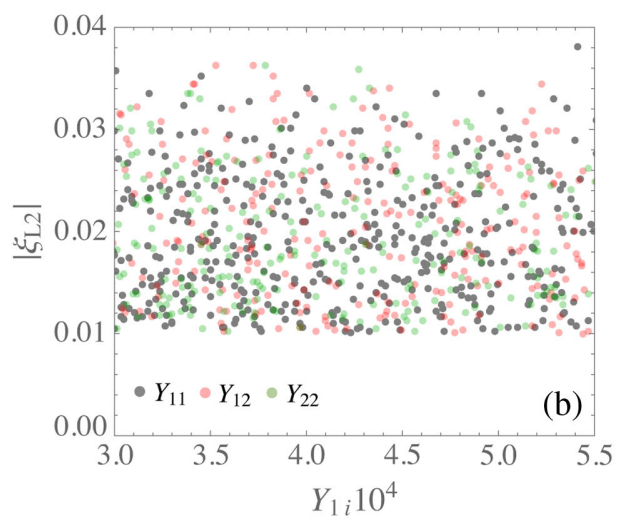

FIG. 5. Correlation between $\left|\xi_{L 2}\right|$ and $Y_{i^{\prime} j^{\prime}}\left(Y_{1 i}\right)$, where $i^{\prime}\left(j^{\prime}\right)=2,3 ; i=1,2,3 ; \zeta_{h}<0.3$ and the ranges of $Y_{i j}$ in Eq. (45) are satisfied. 


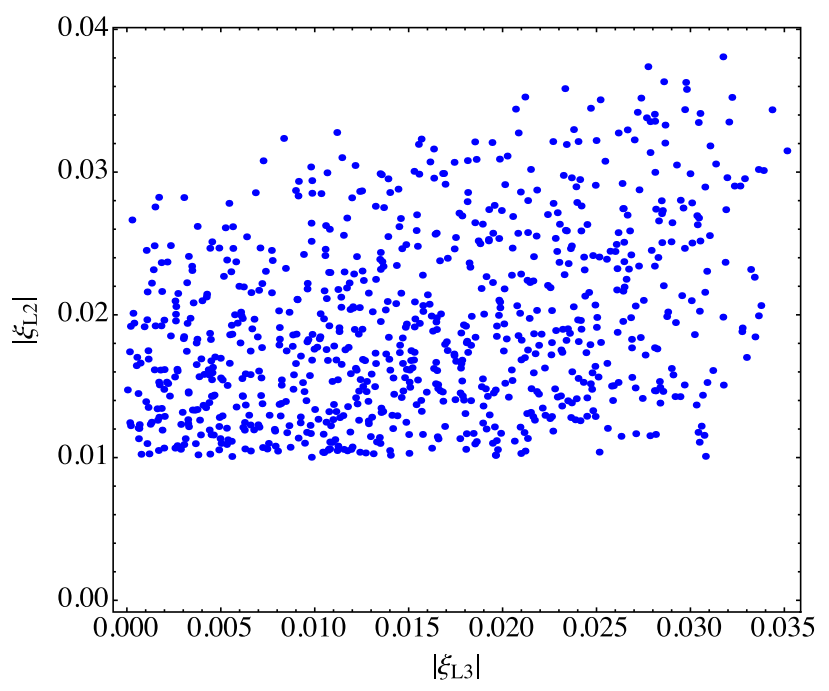

FIG. 6. Correlation between $\left|\xi_{L 2}\right|$ and $\left|\xi_{L 3}\right|$.

$\left|\xi_{L 3}\right|<0.01,\left|\xi_{L 2}\right|$ can still reach 0.03 . Hence, $\operatorname{BR}(\tau \rightarrow \mu \gamma)$ can be well controlled in the model.

\section{B. $\mu \rightarrow e \gamma$ and $\mu \rightarrow 3 e$}

According to the indication shown in Eq. (42), we have to take a small $\xi_{L 1}=0$ to fit the BR for $\mu \rightarrow e \gamma$. For simplicity, $\xi_{L 1}=0$ is fixed in the parameter scan. From Eqs. (23) and (25), it can be seen that even when using $\xi_{L 1}=0$, the BR for $\mu \rightarrow e \gamma$ is still dictated by $y_{R 1}^{*} \xi_{L 2}$; that is, the $\mu \rightarrow e \gamma$ also gives a strict constraint on the $y_{R 1}$ parameter. In order to understand how $\operatorname{BR}(\mu \rightarrow e \gamma)$ is sensitive to $\xi_{L 1}, \operatorname{BR}\left(\mu \rightarrow e \gamma\right.$ ) (in units of $\left.10^{-13}\right)$ as a function of $\left|\xi_{L 2}\right|$ is shown in Fig. 7(a), where the allowed parameter spaces are applied; $y_{R 2}=2.5$ is used; and the results for $y_{R 1}=(1.0,0.8,0.5) \times 10^{-4}$ are shown, respectively, in the plot. It can be seen that $\mu \rightarrow e \gamma$ can further exclude some parameter regions if the $y_{R 1}$ values approach

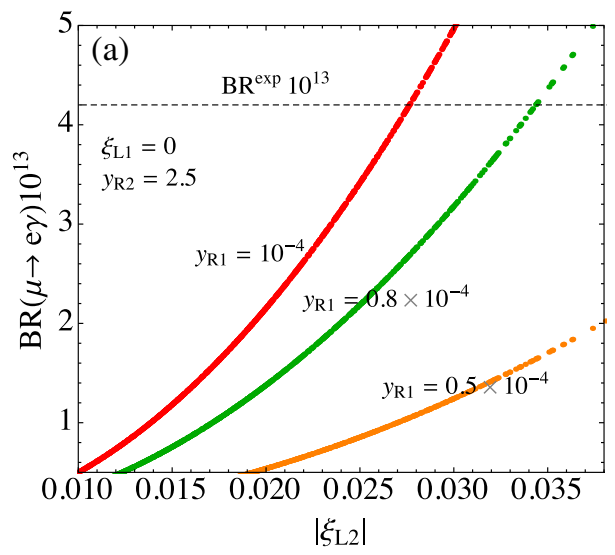

$10^{-3}$ from below. We also show the dependence for the $\mu \rightarrow 3 e$ decay in Fig. 7(b); however, the result is far less than the current upper limit.

\section{C. $\tau \rightarrow \mu \gamma$ and $\tau \rightarrow 3 \mu$}

Using the allowed data points, which are obtained by the parameter scan, we show the scatters of $\operatorname{BR}(\tau \rightarrow \mu \gamma$ ) (in units of $10^{-8}$ ) with respect to $\left|\xi_{L 2}\right|$ in Fig. 8(a), where $y_{R 2}=$ 2.5 and $y_{R 3}=0.5$ are used, and the horizontal dashed line is the experimental upper limit. It can be seen that $\operatorname{BR}(\tau \rightarrow \mu \gamma)$ indeed can further bound the parameter. In order to retain $a_{\mu} \sim 10^{-9}$, we can take a smaller value for $y_{R 3}$. We also show the scatter plot for $\operatorname{BR}(\tau \rightarrow 3 \mu)$ in units of $10^{-8}$ in Fig. 8(b). Although the resulting $\operatorname{BR}(\tau \rightarrow 3 \mu)$ is still smaller than the current upper limit by 1 order of magnitude, the allowed region can still reach the Belle II sensitivity of tau physics.

As mentioned before, $\operatorname{BR}(\tau \rightarrow \mu \gamma)$ depends on the $y_{R 2}^{*} y_{R 3}, y_{R 2}^{*} \xi_{L 3}$, and $\xi_{L 2}^{*} y_{R 3}$ parameters. To gain a better understanding of the correlations among parameters, we show the contours of $\operatorname{BR}\left(\tau \rightarrow \mu \gamma\right.$ ) (in units of $10^{-8}$ ) as a function of $\xi_{L 3}$ and $y_{R 2}$ in Fig. 9, where plots (a) and (b) denote $y_{R 3}=0$ and $y_{R 3}=0.3$, respectively, and $\xi_{L 2}=$ 0.03 is fixed in both plots. From the results, it can be seen that $\operatorname{BR}(\tau \rightarrow \mu \gamma)$ does not vanish at $\xi_{L 3}=0$ when $y_{R 3} \neq 0$. If the Belle II experiment does not find any event for the $\tau \rightarrow \mu \gamma$ decay at the sensitivity of $10^{-9}$ [62], a simple way to suppress the BR for $\tau \rightarrow \mu \gamma$ in the model is to take $y_{R 3}=0$.

\section{Muon $g-2$}

According to above analysis, it is known that the range of $0.01<\left|\xi_{L 2}\right|<0.04$ is allowed in the model. Although we only show the positive values for $\xi_{L i}$, indeed, the same allowed region is also suitable for the negative $\xi_{L i}$ with the exception of the sign. From Eq. (40), it can be seen that $\xi_{L 2}$ and $y_{R 2}$ have to be opposite in sign in order to get a positive

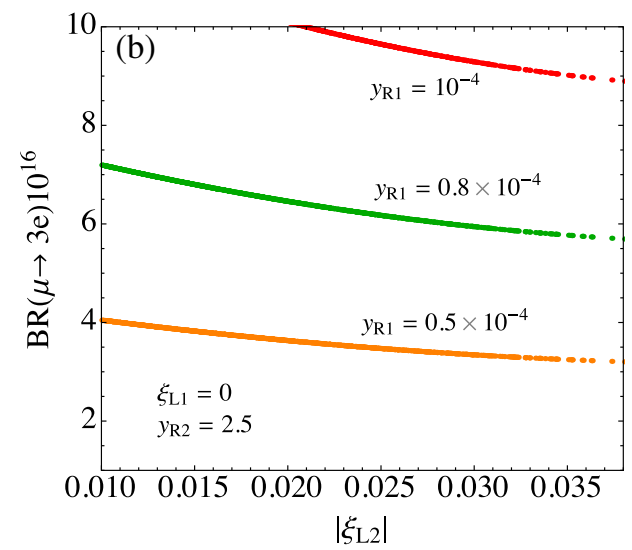

FIG. 7. (a) Scatters of $\operatorname{BR}(\mu \rightarrow e \gamma)$ (in units of $\left.10^{-13}\right)$ as a dependence of $\left|\xi_{L 2}\right|$, where the allowed data points are applied; $\xi_{L 1}=0$ and $y_{R 2}=2.5$ are fixed; and the results with $y_{R 1}=(1.0,0.8,0.5) \times 10^{-4}$ are shown. (b) Scatters of $\operatorname{BR}(\mu \rightarrow 3 e)$ (in units of $\left.10^{-16}\right)$, where the same conditions used in (a) are applied. 

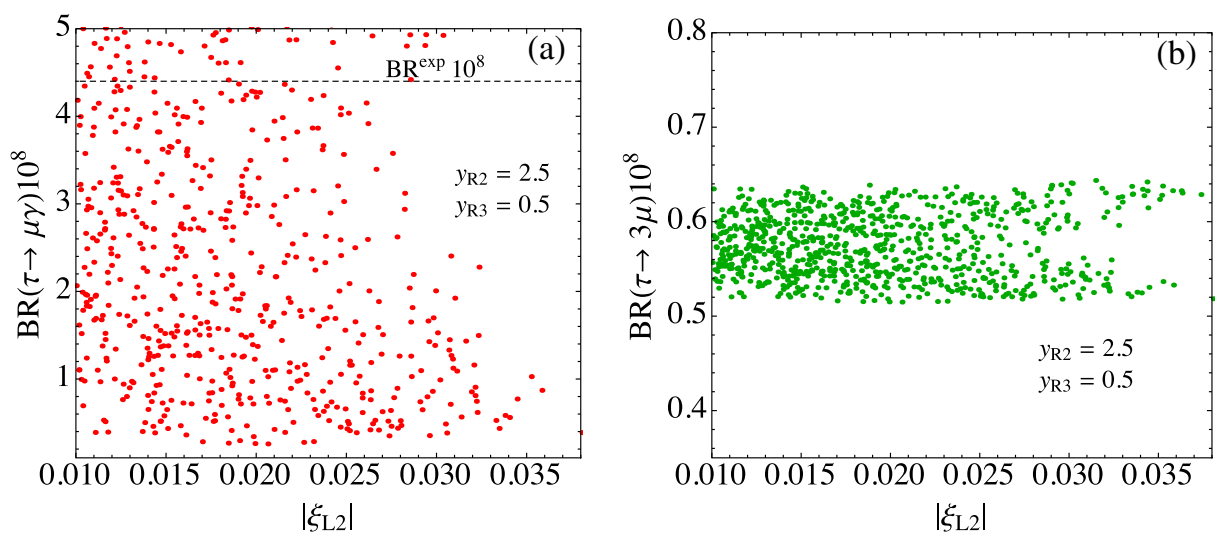

FIG. 8. Scatters for (a) $\operatorname{BR}(\tau \rightarrow \mu \gamma)$ and (b) $\operatorname{BR}(\tau \rightarrow 3 \mu)$, where $y_{R 2}=2.5$ and $y_{R 3}=0.5$ are fixed.
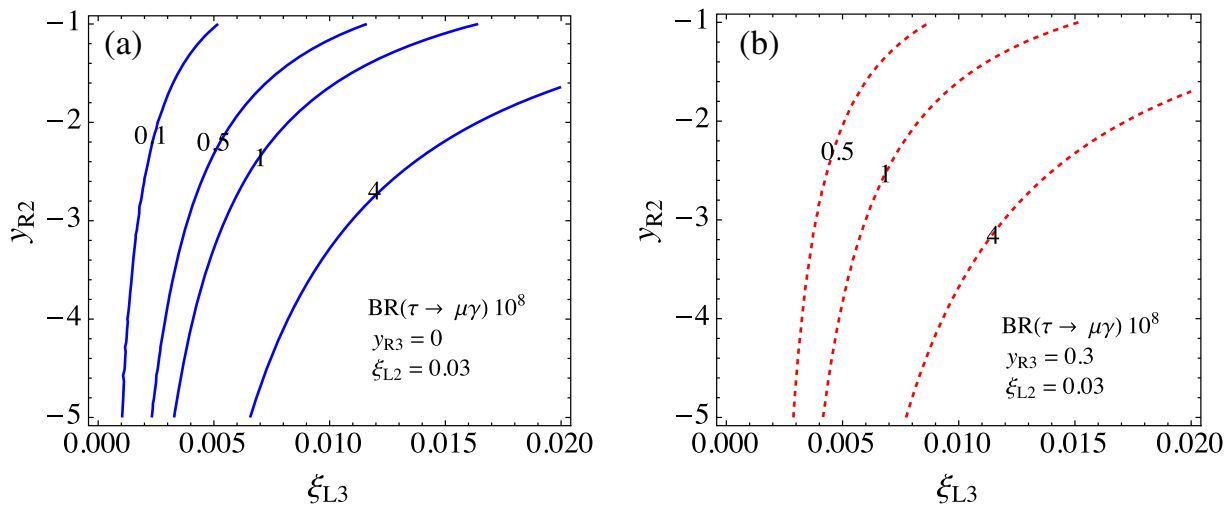

FIG. 9. Contours for $\operatorname{BR}\left(\tau \rightarrow \mu \gamma\right.$ ) (in units of $10^{-8}$ ) as a function of $\xi_{L 3}$ and $y_{R 2}$, where we fix $y_{R 3}=0$ in (a) and $y_{R 3}=0.3$ in (b), respectively. In both plots, $\xi_{L 2}=0.03$ is used.

$a_{\mu}$. To see the influence of inert charged-Higgs-boson effects on the muon $g-2$, we show $a_{\mu}$ (in units of $10^{-10}$ ) as a function of positive $\xi_{L 2}$ and negative $y_{R 2}$ in Fig. 10, where

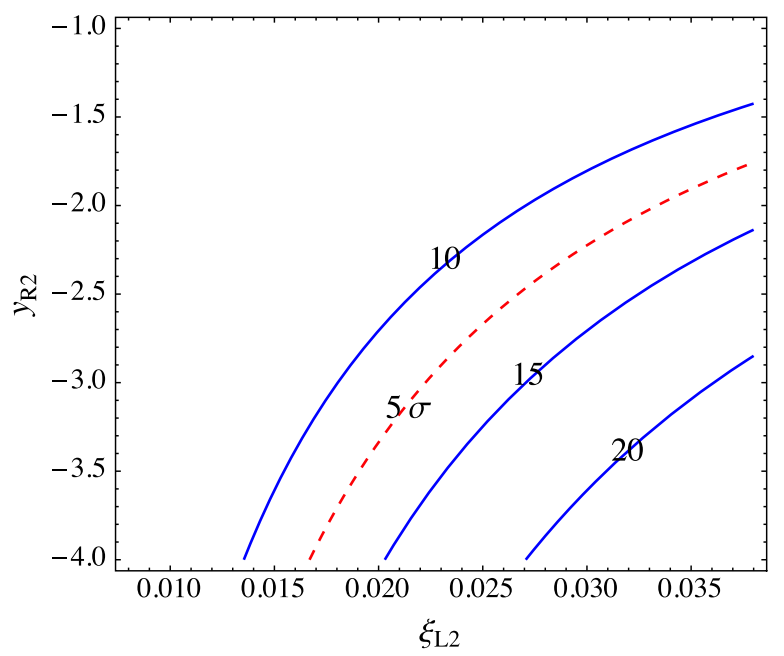

FIG. 10. Muon $g-2$ (in units of $10^{-10}$ ) as a function of $\xi_{L 2}$ and $y_{R 2}$, where the dashed line denotes the $5 \sigma$ result when the experimental and theoretical uncertainties are reduced by a factor of 4 and 2 , respectively. the dashed line denotes the $5 \sigma$ result when the experimental and theoretical uncertainties are reduced by a factor of 4 and 2, respectively. The same result can be applied to the negative $\xi_{L 2}$ and positive $y_{R 2}$. It can be concluded that $a_{\mu} \sim O\left(10^{-9}\right)$ can be realized in the model when the experimental constraints are included.

\section{SUMMARY}

Based on the scotogenic model proposed in [27], we extend the model by including a $Z_{2}$-odd vectorlike lepton doublet $(X)$ in order to resolve the muon $g-2$ excess through the mediation of an inert charged Higgs boson.

In the model, two new Yukawa interactions, i.e., $\bar{X}_{L} H_{I} \ell_{R}$ and $\bar{X}_{L} \tilde{H} N_{k}$, play the key roles. In addition to the new Yukawa couplings, the induced muon $g-2$ also depends on other Yukawa couplings, which are determined by the neutrino mass matrix elements of the order of $10^{-3}-10^{-2} \mathrm{eV}$. It was found that the case with $m_{X, N_{k}}>$ $m_{S_{I}\left(A_{I}\right)}$ cannot significantly enhance the muon $g-2$ because of the bound from the direct dark matter detection. Thus, the suitable dark matter candidate in the model is the lightest $Z_{2}$-odd Majorana lepton. 
Lepton-flavor violation processes, especially $\mu \rightarrow e \gamma$ and $\tau \rightarrow \mu \gamma$, make strict constraints on the relevant parameters. Nevertheless, we found that the resulting muon $g-2$ can reach $O\left(10^{-9}\right)$ when the 11 independent parameter values satisfy the experimental measurements, such as leptonflavor violation, neutrino oscillations, and electroweak oblique parameters. Moreover, the branching ratio for $\tau \rightarrow \mu \gamma$ can be well controlled and can reach the sensitivity of Bell II with an integrated luminosity of $50 \mathrm{ab}^{-1}$.

\section{ACKNOWLEDGMENTS}

This work was partially supported by the Ministry of Science and Technology of Taiwan, under Grant No. MOST-106-2112-M-006-010-MY2 (C. H. C.).
[1] G. W. Bennett et al. (Muon g-2 Collaboration), Phys. Rev. D 73, 072003 (2006).

[2] M. Tanabashi et al. (Particle Data Group), Phys. Rev. D 98, 030001 (2018).

[3] A. Keshavarzi, D. Nomura, and T. Teubner, Phys. Rev. D 97, 114025 (2018).

[4] A. Czarnecki and W. J. Marciano, Phys. Rev. D 64, 013014 (2001).

[5] S. N. Gninenko and N. V. Krasnikov, Phys. Lett. B 513, 119 (2001).

[6] E. Ma and M. Raidal, Phys. Rev. Lett. 87, 011802 (2001); 87, 159901(E) (2001)

[7] C. H. Chen and C. Q. Geng, Phys. Lett. B 511, 77 (2001).

[8] E. Ma, D. P. Roy, and S. Roy, Phys. Lett. B 525, 101 (2002).

[9] R. Benbrik, C. H. Chen, and T. Nomura, Phys. Rev. D 93, 095004 (2016).

[10] T. Nomura and H. Okada, Phys. Lett. B 756, 295 (2016).

[11] S. Baek, T. Nomura, and H. Okada, Phys. Lett. B 759, 91 (2016).

[12] W. Altmannshofer, M. Carena, and A. Crivellin, Phys. Rev. D 94, 095026 (2016).

[13] C. H. Chen, T. Nomura, and H. Okada, Phys. Rev. D 94, 115005 (2016).

[14] S. Lee, T. Nomura, and H. Okada, Nucl. Phys. B931, 179 (2018).

[15] C. H. Chen, T. Nomura, and H. Okada, Phys. Lett. B 774, 456 (2017).

[16] A. Das, T. Nomura, H. Okada, and S. Roy, Phys. Rev. D 96, 075001 (2017).

[17] K. Kowalska and E. M. Sessolo, J. High Energy Phys. 09 (2017) 112.

[18] L. Calibbi, R. Ziegler, and J. Zupan, J. High Energy Phys. 07 (2018) 046.

[19] B. Barman, D. Borah, L. Mukherjee, and S. Nandi, arXiv:1808.06639.

[20] F. Jegerlehner and A. Nyffeler, Phys. Rep. 477, 1 (2009).

[21] J. P. Miller, E. de Rafael, B. L. Roberts, and D. Stockinger, Annu. Rev. Nucl. Part. Sci. 62, 237 (2012).

[22] M. Lindner, M. Platscher, and F. S. Queiroz, Phys. Rep. 731, 1 (2018).

[23] F. Jegerlehner, Acta Phys. Pol. B 49, 1157 (2018).

[24] J. Grange et al. (Muon g-2 Collaboration), arXiv:1501 .06858 .

[25] M. Otani (E34 Collaboration), JPS Conf. Proc. 8, 025008 (2015).
[26] R. Hong (Muon g-2 Collaboration), arXiv:1810.03729.

[27] E. Ma, Phys. Rev. D 73, 077301 (2006).

[28] R. Barbieri, L. J. Hall, and V. S. Rychkov, Phys. Rev. D 74, 015007 (2006).

[29] A. Dedes and H. E. Haber, J. High Energy Phys. 05 (2001) 006.

[30] E. Ma, Phys. Rev. D 98, 091701 (2018).

[31] E. Ma, arXiv:1810.06506.

[32] J. Kubo, E. Ma, and D. Suematsu, Phys. Lett. B 642, 18 (2006).

[33] K. S. Babu and E. Ma, Int. J. Mod. Phys. A 23, 1813 (2008).

[34] G. B. Gelmini, E. Osoba, and S. Palomares-Ruiz, Phys. Rev. D 81, 063529 (2010).

[35] A. Adulpravitchai, M. Lindner, A. Merle, and R. N. Mohapatra, Phys. Lett. B 680, 476 (2009).

[36] D. Schmidt, T. Schwetz, and T. Toma, Phys. Rev. D 85, 073009 (2012).

[37] R. Bouchand and A. Merle, J. High Energy Phys. 07 (2012) 084.

[38] M. Klasen, C. E. Yaguna, J. D. Ruiz-Alvarez, D. Restrepo, and O. Zapata, J. Cosmol. Astropart. Phys. 04 (2013) 044.

[39] A. Vicente and C. E. Yaguna, J. High Energy Phys. 02 (2015) 144.

[40] A. Merle and M. Platscher, Phys. Rev. D 92, 095002 (2015).

[41] A. Merle and M. Platscher, J. High Energy Phys. 11 (2015) 148.

[42] A. Ibarra, C. E. Yaguna, and O. Zapata, Phys. Rev. D 93, 035012 (2016).

[43] A. Merle, M. Platscher, N. Rojas, J. W. F. Valle, and A. Vicente, J. High Energy Phys. 07 (2016) 013.

[44] A. Ahriche, K. L. McDonald, and S. Nasri, J. High Energy Phys. 06 (2016) 182.

[45] M. Lindner, M. Platscher, C. E. Yaguna, and A. Merle, Phys. Rev. D 94, 115027 (2016).

[46] D. Borah, S. Sadhukhan, and S. Sahoo, Phys. Lett. B 771, 624 (2017).

[47] D. Borah and A. Gupta, Phys. Rev. D 96, 115012 (2017).

[48] C. Hagedorn, J. Herrero-Garcia, E. Molinaro, and M. A. Schmidt, J. High Energy Phys. 11 (2018) 103.

[49] S. Baumholzer, V. Brdar, and P. Schwaller, J. High Energy Phys. 08 (2018) 067.

[50] D. Borah, P. S. B. Dev, and A. Kumar, Phys. Rev. D 99, 055012 (2019).

[51] S. Bhattacharya, P. Ghosh, N. Sahoo, and N. Sahu, Front. Phys. 7, 80 (2019). 
[52] Q. H. Cao, E. Ma, and G. Rajasekaran, Phys. Rev. D 76, 095011 (2007).

[53] D. Aristizabal Sierra, J. Kubo, D. Restrepo, D. Suematsu, and O. Zapata, Phys. Rev. D 79, 013011 (2009).

[54] S. Bhattacharya, N. Sahoo, and N. Sahu, Phys. Rev. D 93, 115040 (2016).

[55] A. G. Hessler, A. Ibarra, E. Molinaro, and S. Vogl, J. High Energy Phys. 01 (2017) 100.

[56] M. A. Díaz, N. Rojas, S. Urrutia-Quiroga, and J. W. F. Valle, J. High Energy Phys. 08 (2017) 017.

[57] A. Ahriche, A. Jueid, and S. Nasri, Phys. Rev. D 97, 095012 (2018).

[58] E. Boos and I. Volobuev, Phys. Rev. D 97, 095014 (2018).

[59] A. Ahriche, A. Arhrib, A. Jueid, S. Nasri, and A. de La Puente, arXiv:1811.00490.

[60] H. Cai, T. Nomura, and H. Okada, arXiv:1812.01240.
[61] T. Toma and A. Vicente, J. High Energy Phys. 01 (2014) 160.

[62] T. Aushev et al., arXiv:1002.5012.

[63] M.E. Peskin and T. Takeuchi, Phys. Rev. D 46, 381 (1992).

[64] L. Lavoura and J. P. Silva, Phys. Rev. D 47, 2046 (1993).

[65] C. Arina, R. N. Mohapatra, and N. Sahu, Phys. Lett. B 720, 130 (2013).

[66] Y. Cai, J. Herrero-García, M. A. Schmidt, A. Vicente, and R. R. Volkas, Front. Phys. 5, 63 (2017).

[67] P. F. de Salas, D. V. Forero, C. A. Ternes, M. Tortola, and J. W. F. Valle, Phys. Lett. B 782, 633 (2018).

[68] E. Arganda and M. J. Herrero, Phys. Rev. D 73, 055003 (2006).

[69] J. Hisano, T. Moroi, K. Tobe, and M. Yamaguchi, Phys. Rev. D 53, 2442 (1996). 